\title{
Diseño e implementación de un prototipo de identificación digital de datos técnicos vehiculares utilizando tecnología inalámbrica RFID
}

\section{Design and implementation of digital ID software for vehicle technical data using RFID wireless technology}

Jesús David Quintero P. ${ }^{[1]}$, José Armando Son R. ${ }^{[2]}$ y Gilber Mauricio Cuadrado T. ${ }^{[3]}$

\begin{abstract}
Resumen
Este articulo tiene como objetivo dar a conocer la elaboración de un software de identificación utilizando tecnología RFID (Identificación por radio frecuencia) que permite la captura automática de datos mediante ondas de radiofrecuencia. Consiste en instalar un lector en cualquier sitio o lugar para agilizar el flujo y generar seguridad vehicular. Para desarrollar este modelo teorizamos conceptos acerca de: funcionamiento, tipos de comunicación, propagación, normas, estándares, e interferencias que originan problemas en la transmisión sistemas como por ejemplo los Ubiquiti rocketm5 que transmiten señal Wifi, entonces al cruzarse estas señales pueden generan interferencias. Siendo necesario establecer estructuras que nos definieran como ha sido el avance en la electrónica aplicada.

El sistema de seguimiento vehicular totalmente automatizado incorpora lectores RFID fijos "CSL" (Converge System Limited) instalados en vías y peajes para uso de autoridades de tránsito, empresas públicas y privadas. Logrando hacer una identificación en el menor tiempo posible.
\end{abstract}

Palabras clave: lector; software; frecuencia; tecnología RFID; electrónica; transmisión.

\begin{abstract}
.
The purpose of this article is to shed light on the development of ID software using RFID technology (Radiofrequency identification) that allows automatic data capture by means of radiofrequency waves. It consists in installing a reader in any location in order to streamline traffic flow and increase vehicle safety. To develop the model, we considered concepts such as: operation, types of communication, propagation, norms, standards, and possible interference caused by transmission systems such as Ubiquiti rocket $\mathrm{m} 5$ that transmits Wi-Fi signals. It is necessary to establish structures to define the advances in the electronics applied.

The automatic vehicle monitoring system incorporates fixed RFID readers "CSL" (Converge System Limited) installed on roads and turnpikes to be used by the transit authorities, and public and private entities. This technology allows such entities to identify the vehicles very quickly.
\end{abstract}

Key words: reader, software, frequency, RFID technology; electronics, transmission.

[1] Magíster en Tecnologías de la información y las comunicaciones. Universidad Surcolombiana, Neiva. Avenida Pastrana Carrera 1. jdavid@usco.edu.co

[2] Ingeniero Electrónico. Universidad Surcolombiana, Neiva. Avenida Pastrana Carrera 1ª. son-rj@hotmail.com

[3] Ingeniero Electrónico. Universidad Surcolombiana, Neiva. Avenida Pastrana Carrera 1ª. gmauriciocuadros@gmail.com

Recibido: 10 diciembre 2014 • Aceptado: 27 abril 2015 


\section{Introducción}

La tecnología en sus últimos años ha dado un gran salto pasando de ser una especialidad a un atributo para todos los campos; la industria, la manufactura, el transporte, las comunicaciones, e Internet han sido participe de ella, favoreciendo la realización de funciones con mayor facilidad y en menor tiempo.

Es sin duda que la tecnología RFID ha experimentado un auge acelerado para la identificación de elementos abarcando intereses y considerándola como un sustituto natural del código de barras, por tal motivo se hace el título de un buen competidor para las diferentes ciencias aplicadas actualmente (Olmeda S, 2008).

Diseño e implementación de un prototipo de identificación digital de datos técnicos vehiculares utilizando tecnología inalámbrica RFID cumple con el propósito de presentar un documento que defina los conceptos sobre este tema (Hernández J, 2007), sino también teorizando definiciones de cómo es su estructura y efectos que tiene sobre la naturaleza de la electrónica mostrando avances que ha tenido durante los últimos años.

Por tal motivo la tecnología RFID en su campo se ocupa en la identificación de elementos por medio de radio frecuencia e inalámbricamente y en la actualidad ha tomado diferentes estructuras de operación: baja frecuencia, alta frecuencia, ultra alta frecuencia, microondas, Wifi y trabaja en diferentes secciones: activa, pasiva y semi-pasiva (Jiménez R, 2012). Las distintas visiones existentes y dimensiones de carácter teórico-práctico derivan de este trabajo la necesidad de crear un software para la identificación de documentos técnicos vehiculares con el uso de esta misma (Méndez, 2007).

El enfoque del presente proyecto está orientado para presentarlo en el ámbito de la ingeniería especialmente en la electrónica y de software para incorporar un conocimiento de esta parte de la tecnología (RFID) en el campus de la Universidad.

El contenido programático que se presenta en este documento no solo resulta general sino básico haciéndolo fundamental para el conocimiento de esta tecnología, es así donde se discutirán algunos conceptos y definiciones de estructuras de lenguajes de programación como el "Visual C\#”, "PHP” (pre procesado de hipertexto), "JAVA", "JQUERY", "HTML" (Lenguaje de marcado de hipertexto) y "SQL Server" (lenguaje de consulta estructurada). Una solución ideal para tal problema es la utilización de tecnología RFID, ya que es económica, puede comunicarse a una distancia considerable, el margen de error es mínimo. RFID permite identificar un objeto determinado en este caso un vehículo (Larretape, 2010). El dispositivo funciona con una etiqueta ("tag") la cual se encuentra previamente ubicada en los vehículos que mediante el uso de un sistema lector y antenas instaladas en los puntos de referencia se puede comunicar con el sistema de procesamiento, que conectado a una interfaz gráfica, mostrara las propiedades relacionadas con el código único del "tag”" ( Benavides, 2009).

El uso de RFID actualmente ha alcanzado objetivos en todos los campos de la tecnología, utilizándose en aplicaciones móviles hasta en el sectores de la medicina utilizándola como seguimiento de personas y uso medicinal, por ello el modelo de este sistema ha motivado que sea de principal prioridad para ampliar nuestros conocimientos sobre este tema, $\mathrm{y}$ viendo en que sectores de la industria ha sido utilizado actualmente, observado los cumplimientos de un modelo teóricopráctico eficiente.

\section{Metodología}

El proyecto elaborado permite identificar un vehículo en particular con el uso de etiquetas RFID y un lector CSL203, con bases de datos realizadas en SQL es posible que conectado a un servidor (local o externo) se pueda realizar la identificación de los ítems deseados de un vehículo (técnico-mecánica, seguro obligatorio, color, placa, comparendos, etc.). Ésta información puede variar de acuerdo a las necesidades de las autoridades pertinentes para cada situación. Con las bases de datos se podría incluso verificar si un carro acaba de ser robado, el cual puede ser localizado al pasar por un lector RFID y tener una respuesta inmediata de las autoridades. También es posible que las autoridades puedan ir avanzando por una carretera urbana o nacional e ir identificando las características de cada vehículo, inclusive sin hacer que se detengan, debido a que el lector puede adquirir la información de un "tag" en tan solo 1 micro-segundo.

El diagrama de bloques del sistema se muestra en la figura 1.

\subsection{Hardware de identificación}

El sistema cuenta con un lector CSL203, un "Switch", un computador, celular inteligente y etiquetas RSI-674 de ultra alta frecuencia (UHF). 


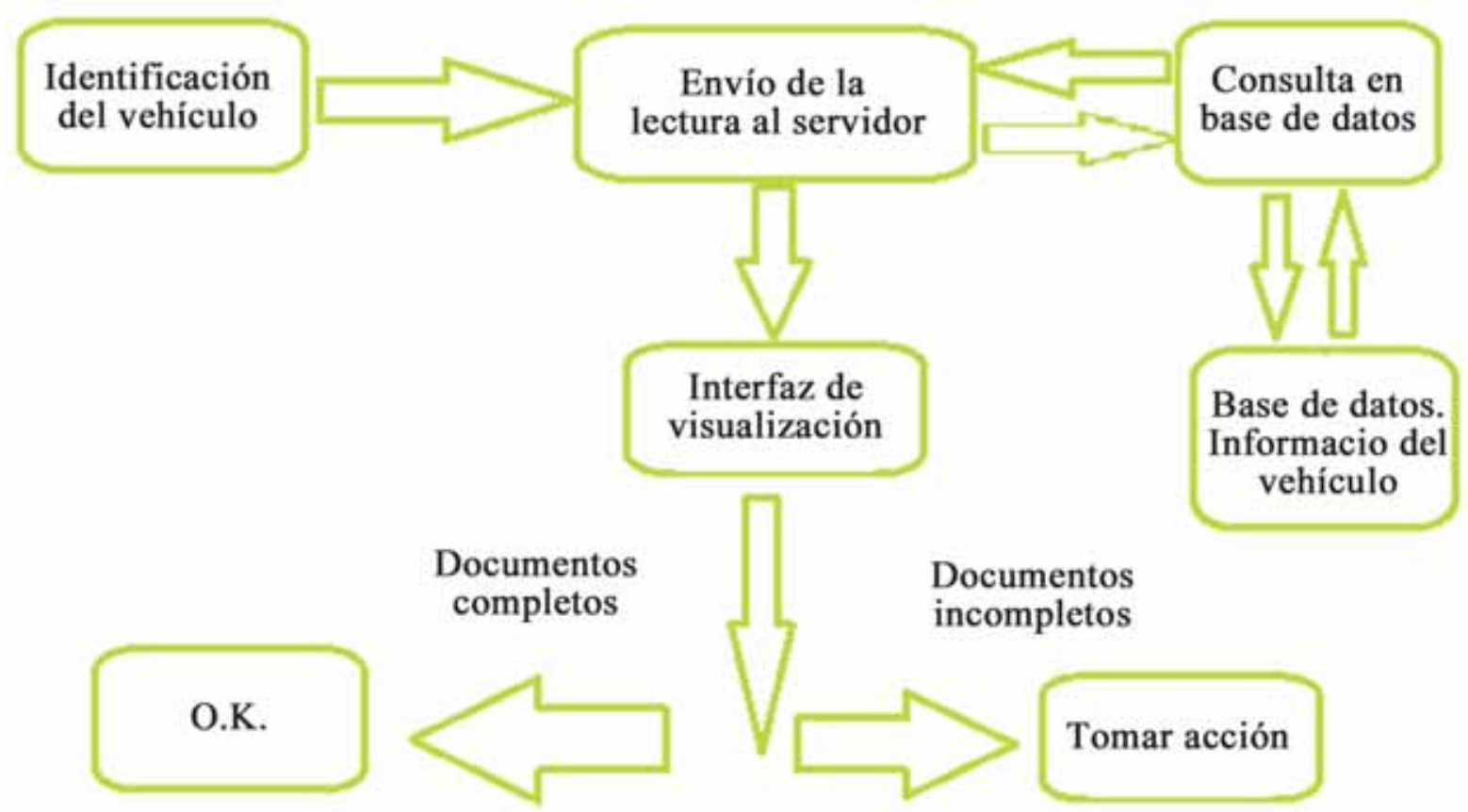

Figura 1. Sistema de identificación vehicular.

2.1.1 Lector. "CSL Converge Systems Limited CS203”. El CSL203 es un lector de RF (Radiofrecuencia) que funciona en el rango de ultra alta frecuencia (800MHz-945MHz); éste lector puede lograr la identificación de una etiqueta en un radio máximo de entre 9 m y 13 m (dependiendo de la etiqueta), si por razón alguna se encuentran más vehículos sobre este rango, automáticamente el lector tomara la primera etiqueta, la mostrará y la guardara en la base de datos del sistema, después toma siguiente visualizándola esta última, puede usarse en una alta variedad de ambientes, soportando temperaturas desde $-20^{\circ} \mathrm{C}$ hasta los $60^{\circ} \mathrm{C}$, con unas dimensiones de tan solo $30 \mathrm{cmX} 30 \mathrm{~cm} X 7,5 \mathrm{~cm}$ en su alto, ancho y grosor respectivamente, que lo hacen muy portable en comparación a otros sistemas con las mismas capacidades. Una de sus ventajas de aplicabilidad para este proyecto es su fácil uso al aire libre.

2.1.2 Switch. Encargado de generar una LAN (red de área local) para que exista comunicación entre el lector CSL203 y un computador.

2.1.3 Etiquetas. Para nuestro desarrollo de trabajo contamos con una etiqueta RSI-674, con características que trabajan bajo una frecuencia UHF, y de un alcance entre 10 a 20 metros.

Características como etiqueta de silicio, tecnología “Impinj Monza”, frecuencia nominal entre 860MHz y
960MHz, memoria EPC de 96 bits y protocolo EPC: UHF clase 1 , gen 2 .

2.1.4 Computador. Principalmente usado para las interfaces de visualización de los datos que lee el equipo RFID, también usado como servidor en el caso que el uso sea local. El diagrama del sistema puede verse en la figura 2.

2.1.5Celular Inteligente. Como se adiciono una etapa para la realización y visualización de comparendos, se tomó la decisión de que esta sea en un aplicativo móvil con la intención de que sea más fácil el acercamiento al vehículo, para mostrarle el procedimiento a realizar.

\subsection{Software}

Para la modelización de los sistemas de software se crea un lenguaje unificado que permite el avance y facilidad de mejorar las metodologías y avanzar en la industria del software.

UML Lenguaje Unificado de Modelado es un lenguaje gráfico para visualizar, especificar, construir y documentar un sistema.

El software fue desarrollado en Visual Estudio 2008 el cual permite la programación en C\# (programación orientada a objetos, basada en $\mathrm{C}$ ), y que a su vez 


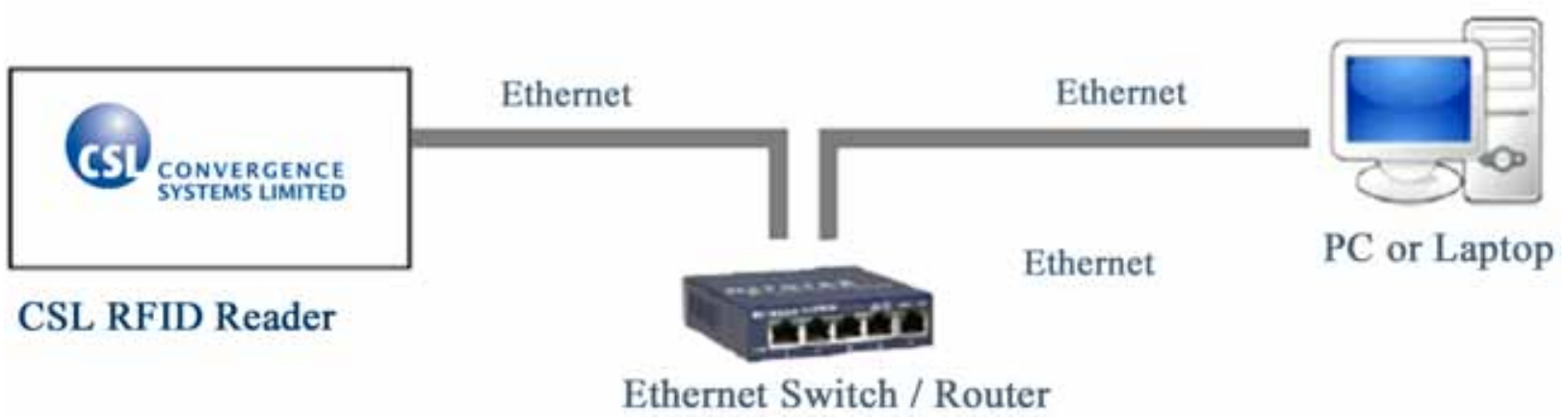

Figura 2. Conexión entre los equipos para el sistema RFID.

permite la conexión con el equipo CSL203, se hizo uso también del motor de bases de datos "SQL SERVER 2008" y su administrador el "SQL SERVER MANAGEMENT ESTUDIO”.

Adicionalmente se desarrolló una interfaz gráfica para dispositivos móviles, haciendo uso de "DREAMWEAVER" el cual permite la conexión con el "PHONEGAP BUILD" y librerías de "JQUERY MOBILE", también se hizo uso de "SUBLIME TEXT", que permiten la programación en "PHP”, "HTML" y por último el uso de "MYSQL" como motor de bases de datos para la página móvil.

2.2.1 Software de identificación. Éste cuenta con una base de datos que contiene los registros y tablas, un administrador que asigne los usuarios para poder acceder al sistema, interfaz de información personal, es decir, datos del propietario del vehículo, sistema de identificación vehicular (datos del vehículo), sistema de categoría del vehículo, si pertenece a una empresa, tipo de vehículo, público o privado y una interfaz para identificar sanciones si el conductor las ha cometido.

2.2.1.1. Quedando el sistema como el mostrado en la figura 3.

Interfaz para ingreso:

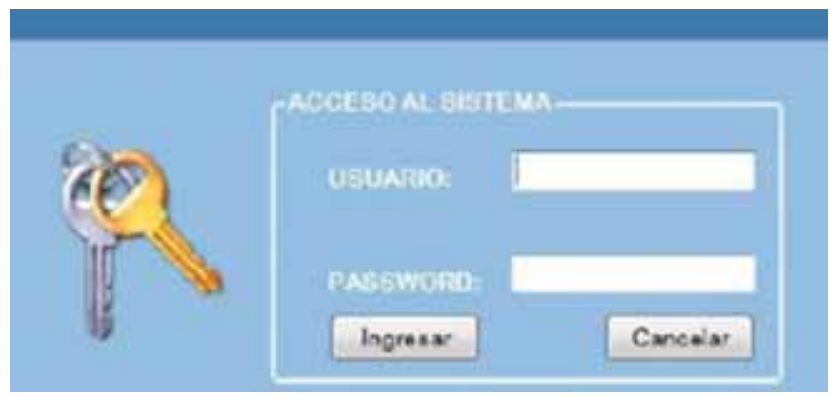

Figura 3. Interfaz para el ingreso. Fuente autores.
Etapa importante en el sistema, con el fin de evitar que cualquier persona pueda ingresar al sistema, solo se puede acceder a través del administrador, quien cuenta con un usuario y una contraseña única, él puede otorgar o crear usuarios pero con el debido consentimiento de que quien lo va a usar es para realizar registros de identificación.

Cuando accedemos al sistema se cuenta con herramientas que ayudan a supervisar y realizar registros vehiculares (mostrado en la figura 4). Con el menú herramientas se puede acceder a las distintas interfaces para los registros del software de identificación RFID.

La siguiente imagen (figura 5), es la interfaz de ingreso de los datos, la cual cuenta con las siguientes características. Datos de registro personales, que son: nombres, apellidos, identificación y número de "EPC" (Código de producto electrónico), con los botones para guardar, eliminar, borrar y editar los datos del propietario.

Se adiciona también una etapa de registro personal, en la cual se ingresan todos los datos del propietario del vehículo (mostrado en la figura 6).

En la figura 7 se dan a conocer todos los datos del vehículo como tarjetas, licencias, certificado, soat, técnico-mecánica, placa, color, modelo, fecha de inscripción, nombre, detalle y una imagen.

\subsection{Desarrollo de la interfaz para la realización de comparendos}

2.3.1. Debido a que el proyecto está enfocado a la identificación de los datos de los diferentes vehículos que pasan por determinada vía, se hace necesario que en el caso de que un vehículo o el conductor no cumplan con los requerimientos de ley, el vehículo será 


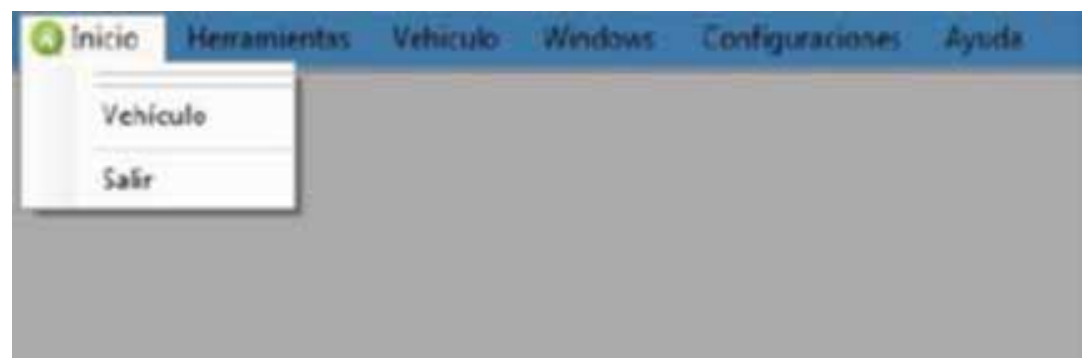

Figura 4. Herramientas del sistema. Fuente autores.

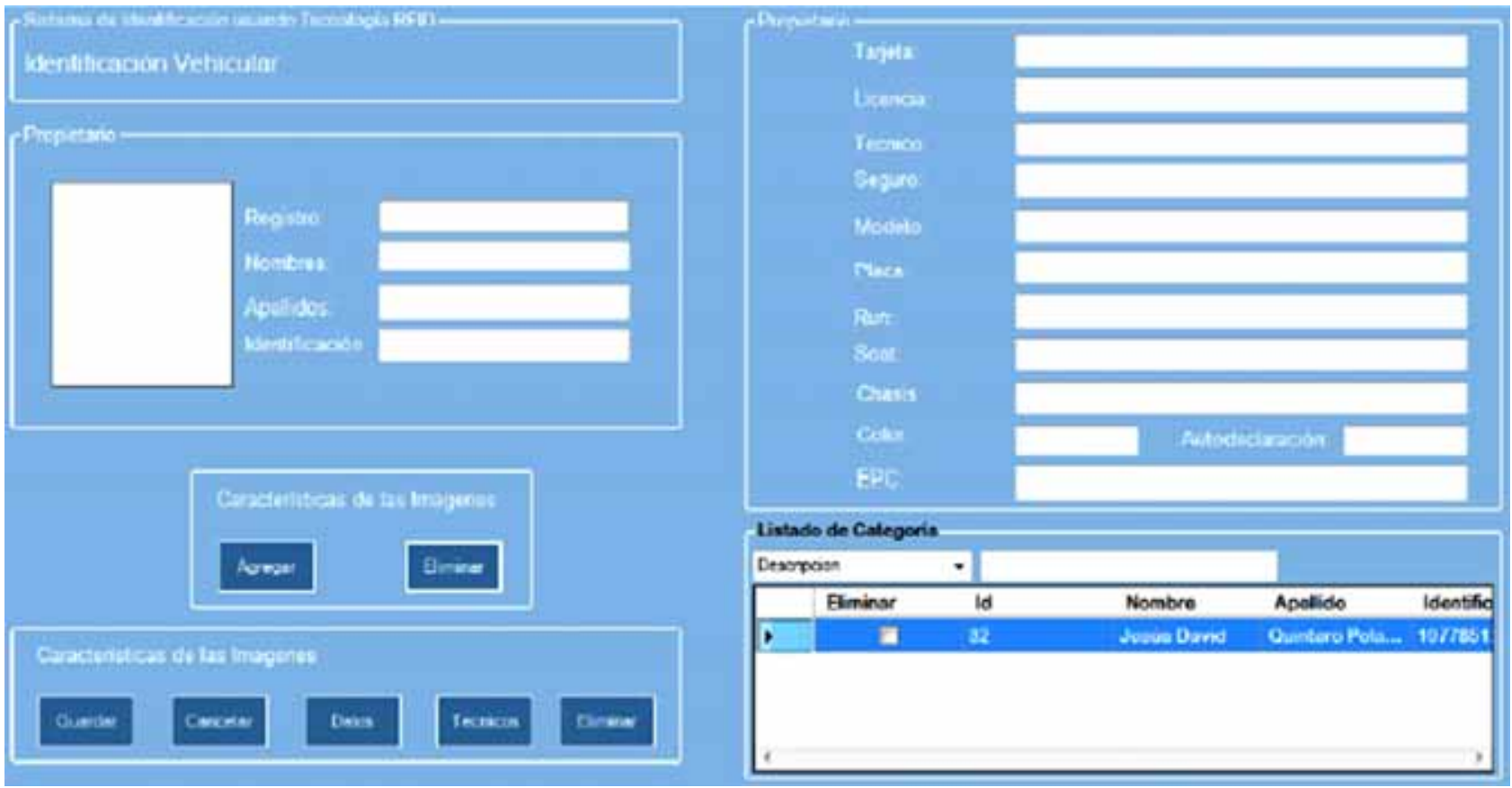

Figura 5. Interfaz para el ingreso de los usuarios. Fuente autores.

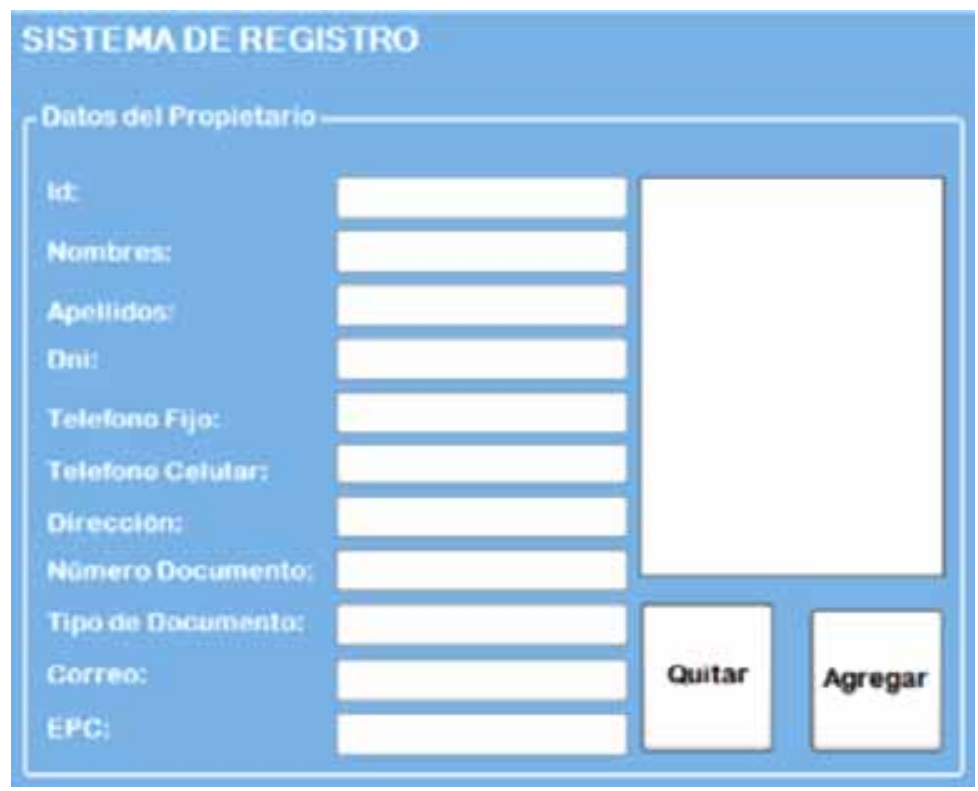

Figura 6. Sistema de registro personal. Fuente autores. 


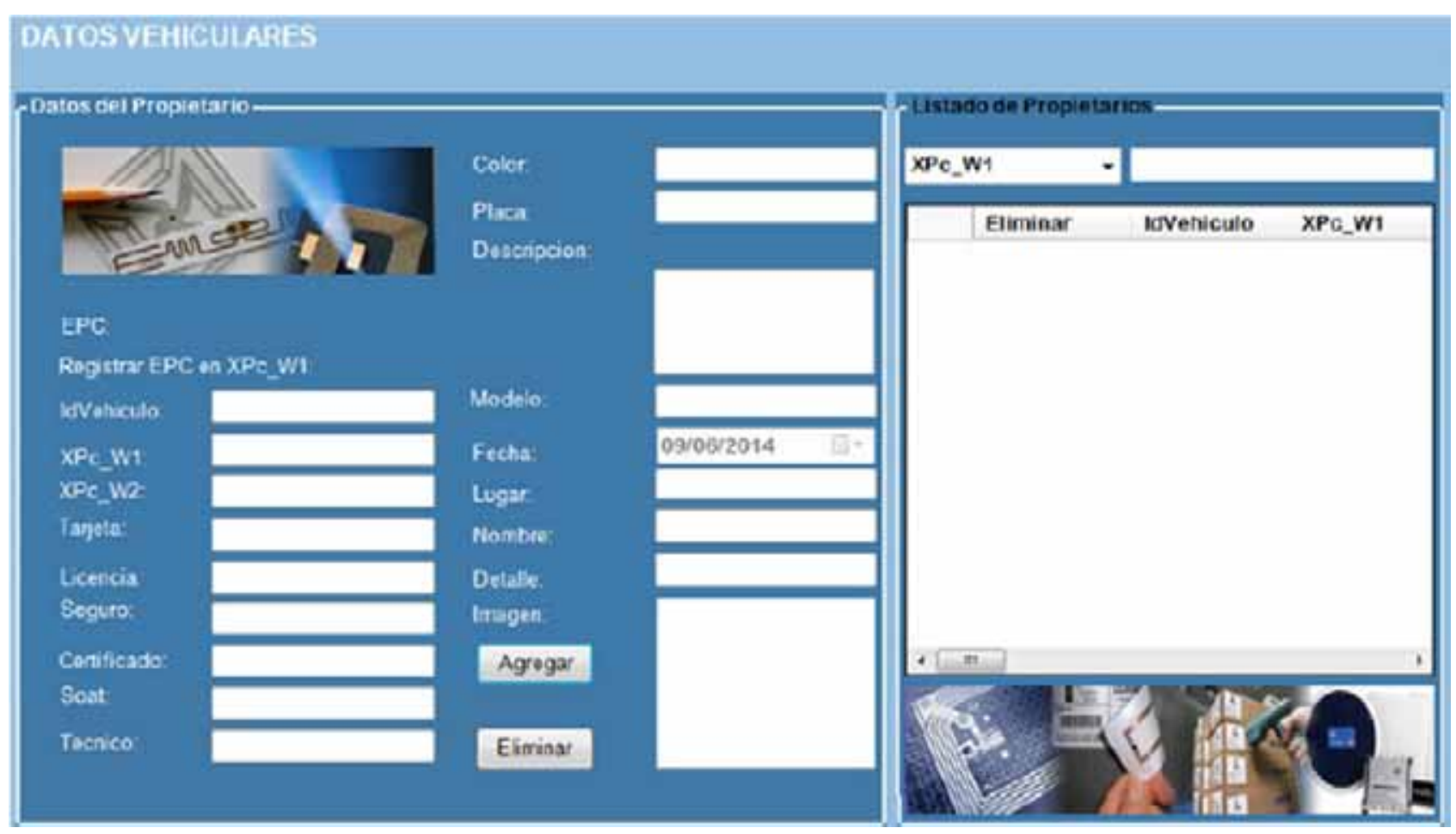

Figura 7. Interfaz para los datos del vehículo. Fuente autores.

retenido o se le realizara una sanción al conductor. Por tanto se asocia al sistema la posibilidad de realizar un comparendo a través de un dispositivo móvil inteligente, lo cual se desarrolló de la siguiente manera.

\subsubsection{1 pasos para el desarrollo de esta interfaz:}

- Se realiza la búsqueda de una plantilla en "DreamWaever" para obtener una presentación liviana, elegante y que se pueda ver en dispositivos móviles inteligentes y computadores personales.

- Una vez obtenida la plantilla sobre la cual se trabajó, se procedió a pasar al "sublime text” para realizar las modificaciones pertinentes al código, éste cambio de herramienta se realiza con la intención de trabajar con HTML y PHP a la vez; sin embargo no se dejó de trabajar en el "dreamweaver”, en él se realizan las hojas de estilos para la página.

- El desarrollo de las bases de datos se realizó con MYSQL, por tanto también se hizo necesario el uso de PHP para relacionar las bases de datos con la página web desarrollada.

- Para relacionar los archivos que se conectaban a las bases de datos fue necesario hacer uso del método "POST" para la transferencia de información confidencial, ya que el método "GET" permite ver la información que se está trasladando de un archivo a otro, junto con el método post se hizo uso del comando "password” en el campo contraseñas, para evitar la visualización de éstas.

- Se carga los archivos en un servidor gratuito, con éste podemos acceder a los datos desde cualquier pc o dispositivo móvil inteligente con una conexión a Internet; esto realizado con el fin de tener unos efectos más reales a la hora de ver el funcionamiento de la página, debido a que con el "wamp server" los datos son cargados de manera inmediata, ya que todo está cargado en el mismo dominio.

- Se realiza la compilación en "PHONEGAP BUILD” para generar el “App” (aplicación móvil) que va a ser instalado en los dispositivos con sistema operativo “Android”, para éste punto se hace necesario crear una interfaz solo con código “HTML”, “CSS” (Hoja de estilos en cascada) y "JQuery Mobile", realizando enlaces desde “HTML” al servidor donde se encuentra alojada la aplicación. 


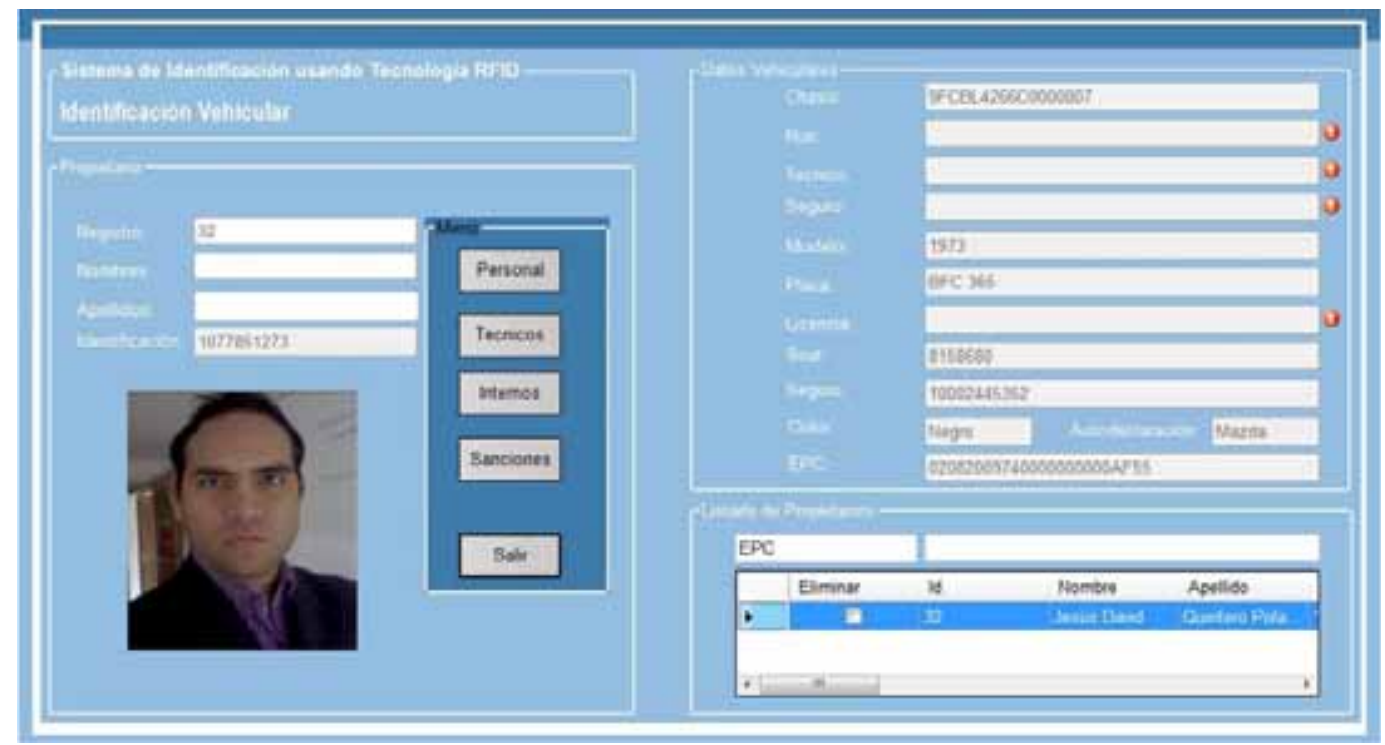

Figura 8. Sistema de registro personal. Fuente: autores.

\section{Resultados}

\subsection{Interfaz de visualización de los datos del vehículo y del propietario}

Se pueden ver los demás datos del vehículo y del propietario, como lo son, datos técnicos del vehículo y sanciones presentadas por el dueño del automotor, en la figura 8 se muestran los datos personales del propietario como nombres, apellidos, DNI, teléfono fijo, teléfono celular, dirección, numero de documento, tipo de documento, correo, “EPC” y una imagen. En la figura se ve un icono rojo el cual representa la falta de alguno de los ítems necesarios para que un conductor esté en regla con la ley.

Cuando el lector captura la variable "EPC” se envía a un formulario donde se encuentra almacenados todos los datos del propietario entonces él hace una búsqueda e identifica quien es el dueño del automóvil y las características del vehículo, llevando esta información a las interfaces de visualización (figura 8).

\subsubsection{Interfaz de visualización para la realización de un comparendo}

La página desarrollada cuenta con un total de 11 páginas anidadas en un mismo archivo PHP, con la ayuda de las propiedades del “jQuery Mobile” podemos tener una interfaz individual para cada página, sin importar que estén en el mismo PHP. La página principal está compuesta simplemente por seis imágenes que llevan a las demás páginas (figura 9).

\section{CONTROL VEHICULAR}

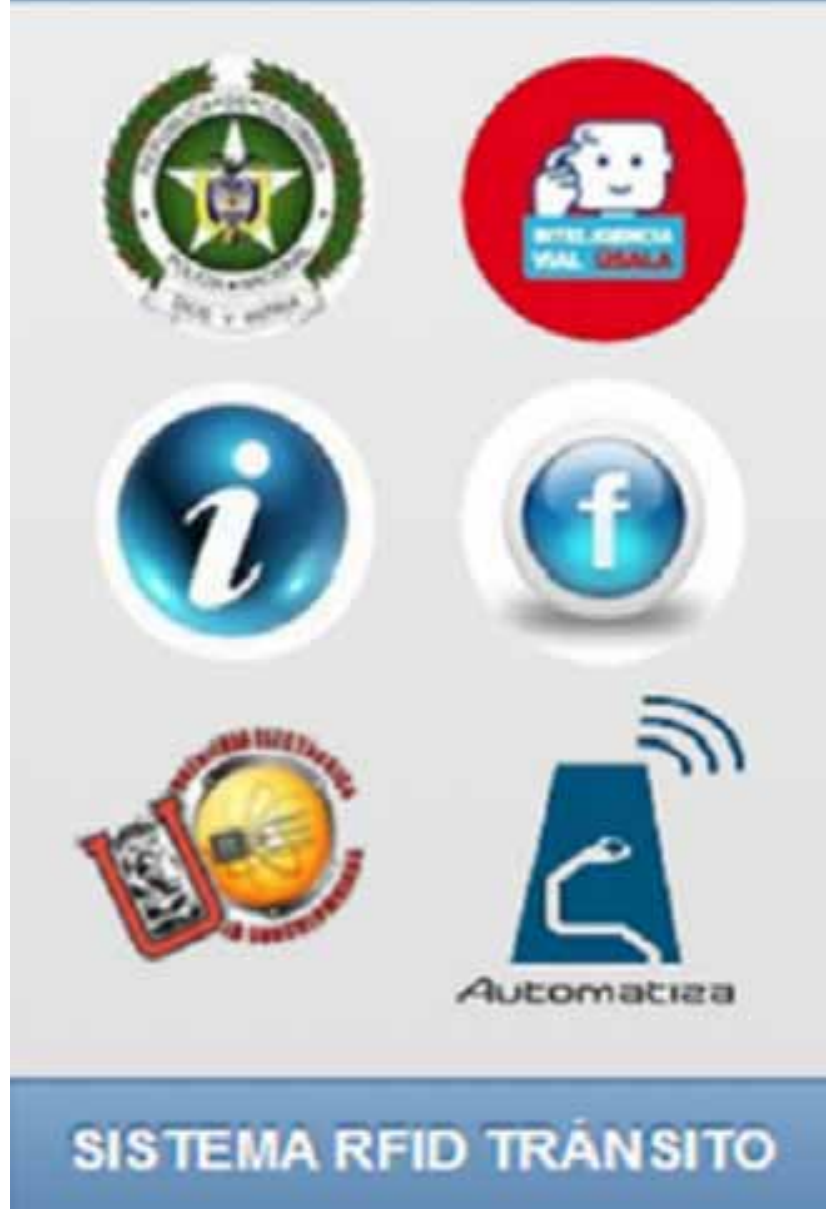

Figura 9. Interfaz del sistema visto desde un computador. Fuente: autores. 
En cada una de ellas se encuentran botones para la operación deseada, y un botón adicional para el regreso a la pantalla principal, a continuación en la figura 10 se muestra la interfaz para la realización de un comparendo.

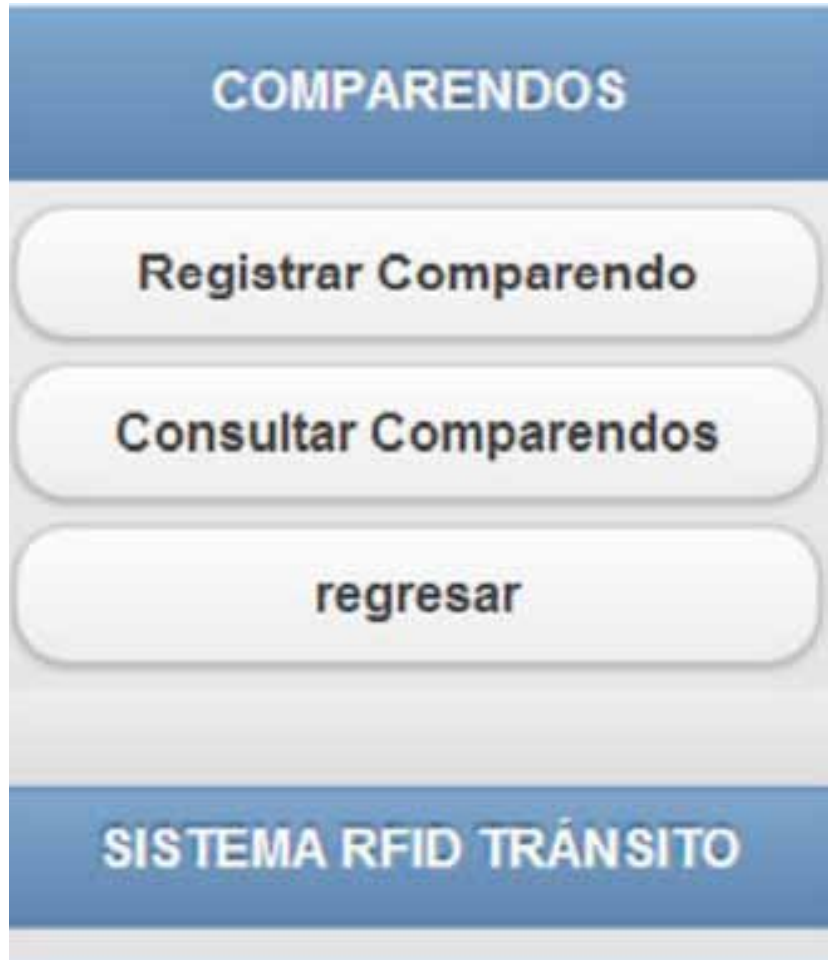

Figura 10. Interfaz para la realización de un comparendo. Fuente: autores.

\section{Conclusiones}

- Se generó una página web que pudiese verse desde cualquier celular inteligente o pc con acceso a internet, logrando una total versatilidad a la hora de visualizar la página. Además de esto, se generó un aplicativo móvil para dispositivos con sistema operativo “Android”, con la intensión de realizar un acceso más fácil y rápido al servidor donde se encuentran alojados los datos, sin embargo esto no es una limitante para los demás equipos que no poseen sistema operativo “Android”, si colocan la dirección correcta del servidor en su navegador, también pueden acceder a los datos, ya sea una persona cualquiera o un agente de tránsito, el aplicativo también quedo para que cualquier persona pueda ver desde su casa si tiene o no comparendos, (teniendo en cuenta que al ser un delito contra la ley puede ser de acceso público), la plataforma nos brinda la posibilidad de ingresar solamente a la revisión de los datos guardados en las bases de datos del sistema, a diferencia de los agentes de tránsito que si podrán realizar la inserción de datos en ellas mismas, ya que a ellos se les asignaría una clave y un usuario para el acceso a la adición de información a las bases de datos, además de que estos mismos tienen la posibilidad y la obligación de eliminar las sanciones una vez canceladas.

- En el caso de implementar un sistema como este se logra un impacto positivo ambiental, ya no será necesario el uso de papel para la realización del comparendo.

- Se logra una mejora en el sistema, el comparendo quedara cargado inmediatamente en los servidores y de esta manera tanto el usuario como el agente podrán verlo en la red de inmediato; logrando que si una persona no paga un comparendo se pueda saber con solo escribir su cédula cuantos comparendos tiene y los diferentes motivos por los cuales se le realzaron los comparendos.

- Para lograr este proyecto se tuvieron en cuenta las etapas que fueron determinantes para su fin, se llevó a cabo que el avance de la Tecnología RFID, es cada vez mayor aún y su desarrollo ha hecho migra de algunos sistemas como el código de barras a este tipo de identificación.

- Los conceptos de comunicación, líneas y antenas, fueron de gran ayuda ya que llevo a cabo de una teoría a algo experimental con el lector, es decir, se pudo comprobar algunas definiciones a partir del módulo.

La identificación por RFID más la vinculación de la programación en diferentes lenguajes llevamos a cabo la construcción de prototipo de identificación de documentos vehicular, además de una plataforma móvil, para demostrar que se puede realizar comparados desde diferentes dispositivos móviles.

- Los modelos de identificación RFID cuentan con un sistema de encriptación que permiten mayor seguridad.

\section{Referencias bibliográficas}

1. Alomair, B. and Radha Poovendran. "Privacy versus Scalability in Radio Frequency Identification Systems.” Computer Communication, Elsevier. 2010. 
2. EPCGlobal Inc. "EPC Radio-Frequency Identity Protocols Class-1 Generation-2 UHF RFID Protocol for Communications at $860 \mathrm{MHz}-960 \mathrm{MHz}$ Version 1.0.9”. Enero 2011.

3. ETSI. "EN 300220 ERM: Electromagnetic compatibility and Rado spectrum Matters(ERM);Short Range Devices (SDR) Radio equipment to be used in the $25 \mathrm{MHZ}-1 \mathrm{GHz}$ frequency range with power levels up to $500 \mathrm{~mW}$. Part 1: Technical characteristics and test methods".

4. Finkenzeller, K. "RFID Handbook: Fundamentals and Applications in Contactless Smart Cards and Identification”. John Wiley \& Sons (May, 2012).

5. Hozak, K, Collier, David A, "RFID as an Enabler of Improve Manufacturing Performance,” Decision Sciences, Volume 39, Number 4, November 2008.

6. ISO. "ISO/IEC 18000-6: Information technology automatic identification and Data capture techniques - Identificación por radiofrecuencia (RFID) a nivel de artículo - Parte 6: Parámetros de comunicación del interfaz aire a 860 - 960 MHz”.
7. Li Lu, Jinsong Han, Lei Hu, Yunhao Liu, and Lionel $\mathrm{MNi}$, "Dynamic Key-Updating: Privacy- Preserving Authentication for RFID Systems", in IEEE PerCom 2012, White Plains, NY, USA, March 2012.

8. López, C “Aplicaciones de sensado: emulación de RFID activo y medición en tiempo real” trabajo final de carrera, 29 de enero 2006.

9. Rao, S, J. Wang, W.-D. Huang, H.F. Tibbals, S.-J. Tang, S.J. Spechler, H. Stephanou, and J.-C. Chiao, T. Ativanichayaphong, Development of an implanted RFID impedance sensor for detecting gastroesophageal reflux, In Proceedings of IEEE International Conference on RFID, Grapevine, TX, March 26-28, pp. 127-133.

10. Shorey, R. Ananda, "Winrfid: A middleware for the enablement of radiofrequency identification (rfid) based aplications," Mobile, Wireless, and Sensor Networks: Technology, Applications, and Future Directions, pp. 313, 336, 2010. 
